\title{
„Alle lebten von Fluchtgedanken“ Gedächtnis und Identität in Herta Müllers Herztier
}

\author{
László V. Szabó
}

,Everyone wished to flee...'

Memory and Identity in Herta Müller's Herztier

\begin{abstract}
The paper argues that Herta Müller's novel Herztier (1994) can be regarded in a certain intercultural sense as an "erratic block" (term used by Kimmerle also 1994), as the historic period constituting the background of the novel, namely, the Ceauşescu-dictatorship in Romania before 1989, may appear from the perspective of today's reader as strange as an African culture, or that of mediaeval Europe. Müller's novel also proves how different the history and culture of certain European regions can be from one another. Moreover, cultural and mental divergences can prevail and endure also after crossing political borders as experienced by Herta Müller when leaving Romania for Germany in 1987. Finally, the paper discusses questions of cultural memory and space, looking for traces of a poetics of memory (also expressed in the motif of flight) in Herztier.
\end{abstract}

Keywords: identity; erratic block; cultural memory; (inter)cultural space; poetics of memory; political, cultural, and mental borders; topography

Schlüsselwörter: Identität; erratische Blöcke; Kulturgedächtnis; interkultureller Raum; Gedächtnispoetik; politische, kulturelle und mentale Grenzen; Topographie

Subject-Affiliation in New CEEOL: Language and Literature - Studies of Literature - German Literature

DOI: $10.36007 /$ eruedu.2021.3.076-087

Heinz Kimmerle stellte einmal, zwar von der Philosophie Afrikas ausgehend, die Anforderung an den Leser, der sich zu einem interkulturellen Verstehen befugt fühlt, man müsse die Sprache, in der sich „das Ganze der Fremdheit“ zwischen Menschen auftut, erfassen, und immer das Wort finden, mit dem man den anderen erreicht. Beim Versuch eines interkulturellen Verstehens bleiben zwar stets „erratische Blöcke“ erhalten, „die sich jeder Einordnung entziehen“, doch solle man im interkulturellen Diskurs den „dialogische[n] Ansatz“ und die „Methodologie des Hörens" beibehalten. Als ein adäquates Mittel zu einem interkulturellen Verstehen nennt Kimmerle das „Hören“, das „gelernt sein“ wolle und „Offenheit, Konzentration, Disziplin und eine methodisch geleitete Technik" erfordere. (Kimmerle 1994, 127) Man übertreibt indessen keineswegs, wenn man behauptet, dass die Intensität des Hörens und der Konzentration nicht nur bei der Begegnung mit den 
Texten einer so (anscheinend) fremden Kultur wie die afrikanische notwendig sind, sondern auch beim Lesen von Texten, die zwar in Europa geschrieben wurden, für manche Leser dennoch so fremd anmuten können, als ob sie auf einem unerreichbar fremden Kontinent entstanden wären. Rumänien zur Zeit der Diktatur Ceauşescus war und ist an sich eine fremde Welt, die mangels einer besonderen Offenheit des Lesens ebenso verschlüsselt bleiben kann wie ggf. ein Stück afrikanischer Literatur. Rein geographisch gesehen war Rumänien der siebziger-achtziger Jahre, in dem sich mehrere Romane Herta Müllers abspielen, ein Teil des modernen Europas - was aber seine Gesellschaft, seine Politik, seinen Geist anbelangt, so war es wohl noch dunkler als das schwarze Afrika oder das europäische Mittelalter. Leser, die diese Welt nicht erlebt haben, werden mit Befremdung solche Romane wie Herztier (Müller 1994) ${ }^{1}$ lesen, schlimmstenfalls könnte ihnen der ganze Roman als ein massiver ",erratischer Block“, als ein Schloss ohne Schlüssel vorkommen.

Es ist eine interessante Sache mit den „erratischen Blöcken“, seien sie literarischer, kultureller oder politischer Natur: Man findet sie überall, wo eine Kultur die andere, eine Zeit die andere, eine Politik die andere nicht versteht, wo kulturelle, räumliche, zeitliche, mentale, diskursive Grenzen und Differenzen vorherrschen, wo das oft beschworene ,Eigene' den Blick auf das ,Andere‘ verdeckt. Man ist beispielsweise im heutigen Europa geneigt, in einem einheitlichen Europa zu denken, von kulturellen Unterschieden abzusehen und kulturelle, wirtschaftliche und sonstige Maßstäbe zu setzen, die in manchen Ecken Europas ziemlich fremd wirken, wenn nicht auf Widerstand stoßen. Geleitet von politischen Kohäsionsdesideraten vergisst man gerne die kulturellen Unterschiede zwischen einzelnen Teilen des Kontinents, die ihrerseits aus unterschiedlichen Traditionen und geschichtlichen Erfahrungen stammen, und man wundert sich, wenn bestimmte Lösungsrezepte nicht überall gleich wirksam sind.

Kulturelle Differenzen hat es in Europa immer gegeben, am meisten wohl zur Zeit der ideologischen Aufteilung Europas, unter der die Völker Südosteuropas Jahrzehnte lang zu leiden hatten. Die Deutschen haben auf eine besondere Weise die Geschichte Europas in der zweiten Hälfte des 20. Jahrhunderts erlebt, nicht nur wegen der ideologischen Trennung der BRD von der DDR, sondern auch wegen des Schicksals der Deutschen im Karpatenbecken. Will man etwa dem Rumäniendeutschen Hans Bergel Glauben schenken, so hat Südosteuropa ein besonderes Schicksal im Laufe der Jahrhunderte erlebt, geprägt durch eine ständige Angst vor der Übermacht der Eroberer, aber auch durch den Hass gegen den Nachbarn, dieser erklärlich wohl als eine Kompensation für jene. Das gemeinsame Schicksal hat dennoch die Völker dieser Region vereint und einen Typus des südosteuropäischen Menschen geschaffen, der sich von jenem des Westeuropäers vielleicht bis heute unterscheidet. Zum Schicksal der Völker dieser Region, auch der Karpatendeutschen, gehörte nicht zuletzt die Aussiedlung, die sich in Rumänien in den siebziger-achtziger Jahren beachtlich intensivierte, was schließlich fast zum völligen Schwinden der Rumäniendeutschen führte. Die Aussiedlung, gelegentlich die Flucht (von der eben auch Herta Müllers Herztier erzählt) waren zunächst ein Zwang, später ein gefährlicher Wunsch, schließlich ein gewollter Abschied von

1 Im Weiteren zitiert mit dem Kürzel „H“ im laufenden Text. 
einer Region, die ihnen mehr als acht Jahrhunderte lang die Heimat war.

Herta Müller verließ das Rumänien Ceauşescus 1987, zwei Jahre vor einer unvorhersehbaren politischen Wende, die sie aber höchst wahrscheinlich nicht verhindert hätte, selbst nach dem Fall des Diktators Rumänien zu verlassen. Denn, nach der kurzen Euphorie im Dezember 1989, als alle Nationen Rumäniens von Hoffnungen auf eine glückliche Zukunft erfüllt waren, kam die große Ernüchterung. Auch die Rumäniendeutschen mussten ihre Hoffnungen schnell aufgeben, dass das postkommunistische Rumänien ein Land nach ihrem Wunsch werden könnte. Es stellte sich bald heraus, dass das scheinbar demokratisierte Rumänien mit den Fehlern und Sünden seiner Vergangenheit nicht abrechnen konnte, sie sogar fortführte. Folglich nahm ein erneuter Exodus seinen Anfang. „Die Erklärung dafür ist so einfach wie ernüchternd“, bemerkte Hans Bergel in einem Essay, „das gleiche mononationale Staatsverständnis, ja weitgehend auch noch der gleiche nationalistische Assimilationsreflex, die in Rumänien von 1945 bis 1989 die kommunistische Politik als ,Nationalismus in Rot" bestimmten, sind heute ohne die Maske des Marxismus und Kommunismus immer noch entscheidende Triebkräfte." (Bergel 1995, 197)

Mit der Aussiedlung ist aber das Problem der Identität und gar der psychischen Integrität nicht gelöst. Man kann das Gedächtnis nicht so einfach hinterlassen wie die Spielzeuge im Dachboden des Elternhauses. Das Trauma der Geschichte lässt sich durch die Überquerung einer politischen Grenze, beim Anblick eines freien Landes und einer verheißungsvollen Zukunft schwer abstreifen. Identität ändert sich nicht über Nacht durch eine Ausreise in ein gelobtes Land. Selbst wenn die Reise innerhalb desselben Sprachraumes erfolgt, kann man der Identitätsfrage nicht entgehen. Den politischen Grenzen gesellen sich Identitäts- und Mentalitätsgrenzen, die kritische und sogar absurde Situationen produzieren können. Symptomatisch ist diesbezüglich Herta Müllers Bericht über die Umstände ihrer Aussiedlung, das Warten auf den Zug in einem kleinen Grenzbahnhof in Rumänien, das böse Spiel der Polizisten, die die Ausreise im letzten Augenblick doch zu verhindern drohen und den Ausreisenden noch den Satz „Nicht vergessen, wir kriegen euch überall“ nachschicken, den mehrere Tage lang andauernden Verhör in den deutschen Behörden. Es findet eine bizarre „Verwandlung“ statt: „Am Vortag in Österreich noch Dissidentin, galt ich jetzt in Nürnberg als Agentin. " (Müller 2013) Mag sein, dass die Überschreitung einer politischen Grenze mit jener einer Kulturgrenze übereinfällt, dass man durch eine Bewegung im Raum sogar eine zeitliche Grenze überschreitet - insofern das Rumänien Ceauşescus sich auch zeitlich ebenso fern von der BRD befand wie das Mittelalter von der Moderne - , doch sind die Gedächtnisräume anders geartet: Ihre Entstehung ist komplexer, vielfältiger, subjektiver als jene der geopolitischen Räume, ihre inneren Grenzen, wenn es sie überhaupt gibt, sind verschwommen, sind mal leichter, mal aber schmerzhafter zu überqueren. Vereinfacht gesagt: Man kann ein Land verlassen, aber man schleppt seine Erinnerungen mit sich, wie ein schweres Gepäck, das man nie mehr loswerden kann. Herta Müller sagte einmal: „Beschädigungen, das muß man sich eingestehen, sind und bleiben Bindungen - notwendig, ungestüm und gnadenlos.“ (Müller 2011, 36) Die Last des Gedächtnisses schreit nach Ausdruck, der „Phan- 
tomschmerz im Erinnern“ (ebda.) will sich artikulieren, sonst droht er, die Seele zu zerreißen. Herta Müller schreibt auch im Namen einer vergessenen Generation, einer vergessenen Zeit, im Namen all derer, deren dumpfe, aber verzweifelte Stimme wegen der Ferne ungehört blieb. Für Viele, die in einer scheußlichen Diktatur unbemerkt verschwunden sind, ist es wohl zu spät. Doch bleibt zumindest der Trost des Gedächtnisses, das schriftliche Denkmal für die Namenlosen, ein spätes Pendant zu den Ungerechtigkeiten der Geschichte.

\section{II.}

Herta Müllers Romane sind Zeugnisse eines von der Last der Geschichte und der schmerzvollen Erinnerungen geplagten Gedächtnisses, in denen mehr oder weniger indirekt auch Fragen nach der Identität einer ihrer Herkunft nach rumäniendeutschen Autorin aufkommen lassen. Romane wie Herztier realisieren eine Poetik des geschichtlichen Traumas und der Fremdheit, die stellenweise an eine Poetik des Schweigens grenzt. Sie fordern zu interkultureller Verständnisoffenheit und geschichtlichem Einfühlungsvermögen auf. Ihre Mnemotechnik dient, über ihre poetische Funktion hinaus, einer Vergangenheitsbewältigung im Sinne jenes Satzes von Herta Müller: „Dieses Land trieb Hunderttausende ins Exil. Wir sollten uns daran erinnern." (ebda.) Geschichte wird im Roman zur Poetik, ${ }^{2}$ zu einer besonderen, verhaltenen Staccato-Sprache, die den Fluss des Erzählens immer wieder unterbricht bzw. die Erinnerungen in verschiedene Richtungen lenkt. Das Narrativ wird grundsätzlich aus einer Kindheits- bzw. Jugendperspektive erzählt, ergänzt noch durch die spätere Perspektive einer Migrantin. Die lose verknüpften Erinnerungsmomente ergeben ein mosaikartiges Erzählen, das ein freies Bewegen auf der chronologischen Achse der Narration erlaubt, indem es den Eindruck der Gleichzeitigkeit des Erzählten erweckt: Die Bruchstücke der Vergangenheit werden gleichsam auf einen weiten „Schirm“ des Gedächtnisses projiziert, der ihren chronologischen Unterschied aufhebt. Vergangenheit wird damit in der Gleichzeitigkeit des Erinnerns gegenwärtig. ${ }^{3}$

Die Kunst Herta Müllers ließe sich nur schwer als „realistisch“ bezeichnen; sie schöpft zwar ihren Erzählstoff aus der erlebten Geschichte Rumäniens bzw. des Banats $^{4}$ in den siebziger-achtziger Jahren des letzten Jahrhunderts, dazu aus autobiographischen Momenten, doch wird das Objektive des einstigen Geschehens im Gedächtnis subjektiviert, gefärbt, poetisiert. Aber von einer Verschönerung der

2 Geschichtspoetik wäre hier wohl ein adäquater Begriff. Dieser meint, dass ein geschichtlicher Wahrheitskern vermöge der dichterischen Phantasie ergänzt, verfärbt, d.h. poetisiert wird.

3 Der Text des Herztier-Romans besteht aus scheinbaren Fragmenten, die einzelne, lose verknüpfte Erinnerungstücke enthalten. Sie zeigen weniger eine chronologische Reihenfolge als eine innere Logik des Erinnerns, die auch zeitlich fernstehende Momente der Vergangenheit nach- oder (gleichsam) nebeneinanderstellt. So folgt beispielsweise auf die Reminiszenz des Kindes, dass ein kleines Rädchen verschluckt, eine Erinnerung an Tereza, die offenbar in einer viel späteren Zeit ,jeden Tag zu mir ins Büro" (H 116) kam.

4 Gemeint ist allerdings jener Teil des Banats, der heute Rumänien angehört, darunter das Komitat Timişoara. Das Banat als „kulturhistorische Transferregion“ umfasst heute Teile von Rumänien, Serbien und Ungarn. Vgl. Höhne (2014). 
Vergangenheit bleibt keine Spur; der verhaltene, fast elegische Ton täuscht über das schonungslose Erzählen einer erlebten Wahrheit nicht hinweg. Die transparente Sprache des Romans lebt aus ihrer Intensität. Symbole und Motive verflechten sich zu einer Gedächtnispoetik, die die erzählte Wahrheit nicht tarnt, sondern, ganz im Gegenteil, mit der Intensität und Suggestivität des Ausdrucks zum Vorschein bringt. Die bedrückende Kulisse der Ceauşescu-Ära, repräsentiert von der omnipräsenten Staatssicherheit - der Securitate und von ihrem Vertreter, Hauptmann Pjele ${ }^{5}$ - , verleiht dem Roman eine besondere Dramatik. Man könnte deshalb von einem dramatischen Roman sprechen, der aus zahlreichen kleineren Szenen besteht, getrennt von kleinen, aber nötigen Atempausen, und verbunden miteinander mit den Fäden der Erinnerung. Daraus die Fragmenthaftigkeit des Romans, dessen einzelne Sequenzen durch Zäsuren voneinander getrennt sind, so wie auch Erinnerungen nicht immer fließend, sondern bruchhaft sind. Manch solche Erinnerungssequenzen bestehen lediglich aus ein paar kurzen Sätzen:

Willst du Kinder, fragte Tereza damals. Nein, sagte ich. Stell die vor, du ißt Himbeeren, Enten und Brot, du ißt Äpfel und Pflaumen, fluchst und trägst die Teile der Maschinen hin und her, fährst Straßenbahn und kämmst dich. Und das alles wird ein Kind. (H 156)

Der Roman versucht das Unsagbare auszusagen, das Unerzählbare zu erzählen, das Verschwiegene zur Sprache zu bringen. Vergangenheitsbewältigung ist keine leichte Aufklärungsarbeit, man weiß, wie viele Autoren nach 1945 sich mit der Erbschaft der nationalsozialistischen Vergangenheit befasst haben, wie viele Jahrzehnte die Kriegserinnerungen in der Literatur fortgewirkt haben und weiterhin wirken. Die Bearbeitung des kommunistischen Erbes von Rumänien wird gewiss auch noch lange dauern; es sind mehrere Völker und damit auch mehrere Literaturen daran beteiligt. ${ }^{6}$ Als Autorin rumäniendeutscher Herkunft befindet sich Herta Müller in einem Zwischenraum, ${ }^{7}$ in dem die Erinnerungen an die rumänische Vergangenheit (und auch an die rumänische Sprache) sich in der Sprache einer Migrantenautorin artikulieren. Ihre Kunst besteht nicht zuletzt in der sprachlichen Vergegenwärtigung einer bedrückenden Vergangenheit, in einer suggestiven Sprache, die sich zum einen aus der Erfahrung eines Minderheitenschicksals in einer der dunkelsten Diktaturen des modernen Europas speist, zum anderen den neuesten Tendenzen der deutschsprachigen Literatur folgt. Herta Müllers Romane sind keine Regionalliteratur, sie gehören, spätestens seit der Verleihung des Nobelpreises, zum Weltkulturgut. Ihre narrative Kunst ist vielfältig, sie ist gleichzeitig deskriptiv und dramatisch, wahrheitstreu und stilisiert. Sie verwendet unterschiedliche Perspek-

5 Der Name entspringt dem rumänischen Wort piele (,Haut'), das auf Oberflächlichkeit, Täuschung oder Unverschämtheit im Sinne des rumänischen Phrasems a fi gros la piele anspielen kann.

6 Um nur ein Bespiel aus der ungarischen Literatur in Rumänien zu nennen: die Tetralogie betitelt Bestiarium Transsilvaniae von Zsolt Láng.

7 Der Begriff ,Zwischenraum“ ist inzwischen sowohl in der Raum-Forschung (nach dem berühmten spatial turn), als auch in der interkulturellen Forschung eingebürgert. Man kann inn verstehen als einen (fiktiven) Raum, den man auch einen interkulturellen Raum nennen kann, und in dem sich Sprachen, Identitäten, Kulturen überlappen, ineinander übergehen und damit eine qualitativ neue, sprachlich-kulturelle Dimension (einen „dritten Raum“) entstehen lassen, so wie im Banat, in dem sich ja auch Herztier größtenteils abspielt. Vgl. Wirth (2012, etwa 23). 
tiven der Narration, die Er- (man sollte vielleicht schreiben: Sie-)Perspektive wechselt mit der Ich-Perspektive, die Kindesperspektive schaltet in einem Atemzug in eine Jugendperspektive um, schließlich werden auch die Erinnerungen an die Auswanderung wach. Die geschichtliche Wahrheit, die den Hintergrund der erzählten Geschichte steht, wird gelegentlich durch Hinweise auf konkrete geschichtliche Namen, wie Hitler oder Ceauşescu, aber auch auf die Nazi-Vergangenheit (den Vater als SS-Soldat) untermauert, am häufigsten werden jedoch die Diktatur bzw. „der Diktator" erwähnt.

\section{III.}

Die Topographie des Erzählens umfasst in Herztier das Dorf, das sich als der Geburtsort der Erzählerin identifizieren lässt, dazu die Stadt Temeswar (rum. Timişoara), in der Herta Müller einst studierte und als Übersetzerin arbeitete ${ }^{8}$ (angedeutet werden etwa der Marktplatz und der Trajansplatz, der Hauptplatz von Temeswar), doch auch der berüchtigte Geburtsort des Diktators, Scorniceşti, gelegentlich noch Ungarn, genauer ,Pest' - der Teil der ungarischen Hauptstadt am linken Ufer der Donau, auch von den Ungarn häufig als Kurzform von Budapest gebraucht -, und natürlich auch Deutschland - konkret Städte wie Köln, Augsburg oder Frankfurt - kommen im Text explizit vor. Die Topographie der erzählten Geschichte zeichnet gleichsam jenen erwähnten, Kulturgrenzen überschreitenden Zwischenraum nach, in dem sich die Geschichte, aber auch Herta Müller selbst bewegt (man denke nur an Ihre Besuche in Rumänien ${ }^{9}$ ).

Der Roman Herztier umfasst einen interkulturellen Raum, der sich vom Banat bis Deutschland erstreckt. Das Banat war schon immer eine Region von mehreren Sprachen und Kulturen, in der Schwaben, Rumänen, Ungarn, Serben, Juden und andere Nationen und Nationalitäten (,,Minderheiten“ inbegriffen), wenngleich nicht immer ohne Konflikte, Jahrhunderte lang zusammenlebten. Man kann hier von einer plurikulturellen Koexistenz sprechen, deren schöner Ausdruck die gemeinsame Beteiligung an jenem Protest gegen das Ceauşescu-Regime in Temeswar im Dezember 1989 war (vgl. Grotzky 2019, 9-53). Von interethnischen Konflikten findet man keine Spur im Roman, ganz im Gegenteil: Schwaben, Rumänen und Ungarn leben hier friedlich miteinander, sie teilen die gleiche Alltagsexistenz, bestimmt durch ihre Beziehung zur Diktatur und ihre praktischen Überlebensstrategien von der Geschäftemacherei bis hin zum Einschmuggeln von Büchern aus dem Ausland. Die Allgegenwärtigkeit der Diktatur und die aus ihr resultierende

8 In einer Episode des Romans wird die Übersetzertätigkeit der Erzählerin geschildert: Als Übersetzerin von Anleitungen für hydraulische Maschinen (wahrscheinlich ins Rumänische) nimmt sie eine Vermittlerposition zwischen zwei, einander fremden Welten ein: der Welt der Wörter und Sprachen auf der einen Seite und der Welt der Fabrikarbeiter und inrer monotonen Arbeitswelt auf der anderen. Die Aussichtslosigkeit eines Lebens in einer sozialistischen Fabrik wird lediglich durch die Hoffnung auf die Flucht gemildert: „So wurden sie alt, wenn sie nicht vorher flüchteten oder umfielen und starben." (H 115)

9 Vgl. etwa ziare.com vom 27.9.2010, im Internet unter: https://ziare.com/cultura/scriitori/herta-muller-romanii-au-sperat-ca-lucrurile-se-vor-clarifica-dupa-caderea-dictaturii-1044621 
permanente Angst bestimmen den Grundton des Romans. ${ }^{10}$ Der Diktator selbst, obwohl nicht persönlich im Handlungsraum des Romans anwesend, ist dennoch ein entscheidender Stimmungsfaktor; ob explizit beim Namen genannt oder mit der pejorativen Kurzform „Tschau“ angedeutet, wird er zur Quintessenz einer unterdrückenden Macht, die das Tun und Denken im Alltag maßgeblich beeinflusst. Die Diktatur ist das radikale Fremde, das befürchtete, unzugängliche, obwohl überall anwesende Andere, das keine Selbstbestimmung und keine Bewegungsfreiheit des Individuums gestattet. Als einzige Widerstandsmöglichkeit bietet sich lediglich die Hoffnung in ihren diversen Formen an: als Hoffnung auf eine tödliche Krankheit des Diktators, die sich aber nie bewahrheitet, oder auf die Flucht ins Ausland, deren geschichtliche Wahrheit in den Grenzgebieten Rumäniens in der Ceauşescu-Ära bekannt ist. Der bedeutungsvolle Satz: „Alle lebten von Fluchtgedanken“ (H 55) erhält im Roman eine motivische Funktion, indem die Flucht wiederholt, in verschiedenen Kontexten auftaucht. Das Pronomen ,alle“ deutet wiederum auf die Allgegenwärtigkeit einer unterdrückenden Macht hin, der jede einzelne Person ausgeliefert war, und vor der zu flüchten (etwa über die Donau, die Grenzfelder, in Güterzügen) die einzige Hoffnung auf eine lebenswürdige Existenz blieb. Das Motiv der Flucht wird überdies mit jenem der Angst ${ }^{11}$ und des Todes, wie etwa im Satz „Jede Flucht war ein Angebot an den Tod“ (H 69), verbunden, was eindeutig die Dramatik, ja die Tragik des Flüchtlingsschicksals akzentuiert. Besonders suggestiv wirkt eine Stelle im Roman, wo die bekannte kommunistische Losung: „Proletarier aller Länder vereinigt euch" im nächsten Satz einem krassen Kontrast begegnet: „Und unten auf dem Boden gingen Schuhe, die das Land in der Flucht verlassen durften." (H 141)

Die Tragik erscheint im Roman auf mehreren Ebenen: als die Tragik einer Schicksalsgemeinschaft in der Diktatur, worauf die immer wieder aufgefundenen Leichen der Fliehenden hindeuten, als der Tod der einzelnen Figuren (Lola, Georg, Tereza, Kurt), und als das dramatische Schicksal der Erzählerin, die, zusammen mit ihren Freunden ${ }^{12}$ Verhöre, Erniedrigungen und Drohungen erleidet. Der Satz „mir pfiff der Tod“ (H 111) betont die innere persönliche Betroffenheit der Erzählerin angesichts des andauernden Zustands lebensgefährdender Bedrohungen. Hauptmann Pjele ist gleichsam die Diktatur in persona, wobei die Figur der Tereza jene graue Zone zwischen Unterdrückten und Unterdückern verkörpert, die sich in der Diktatur in die Beziehungen zwischen Menschen einschob. In der Gestalt des Hauptmanns ist Herta Müller gelungen, durch eine einzige Person den Zynismus und die Skrupellosigkeit der Diktatur darzustellen. Die Reaktion darauf ist die des verhaltenen Schmerzes, bis auf jenen sublimierten Wutausbruch am Ende des Romans, artikuliert in einem beeindruckenden Bild der Empörung und Verzweiflung:

10 Dafür nur ein Textbeispiel: „Sie waren in der Angst zu Hause. Die Fabrik, die Bodega, Läden und Wohnviertel, die Bahnhofshallen und Zugfahrten mit Weizen-, Sonnenblumen- und Maisfeldern paßten auf. Die Straßenbahnen, Krankenhäuser, die Friedhöfe. Die Wände und Decken und der offene Himmel." (H 39)

11 Das metaphorisch variierte Thema ,Angst 'ist im Roman ebenfalls besonders ausgeprägt, was bereits einen der Rezensenten zur Sentenz veranlasste: „Dieser Roman ist die metaphorisch-intuitive Beschreibung einer Angstwelt." Engler (1995, 174)

12 Zur leitmotivischen Funktion der Freundschaft im Roman vgl. Nubert (2014, 219-220). 
Ich wünschte mir, daß Hauptmann Pjele einen Sack mit allen seinen Toten trägt. Daß sein geschnittenes Haar nach frischgemähtem Friedhof riecht, wenn er beim Frisör sitzt. Daß das Verbrechen stinkt, wenn er sich nach der Arbeit zu seinem Enkel an den Tisch setzt. (H 251)

Die konzis und intensiv erzählte Geschichte wird durch ein dichtes Geflecht von Motiven, Metaphern und Symbolen - selbst surrealer Art, wie z.B. Nubert darauf hingewiesen hat ${ }^{13}$ - poetisiert. Das beste Beispiel hierfür ist wohl das Titelsymbol des Herztiers, das in ein durch die Willkür des Blickes, surreal anmutendes Bild eingebettet wird. Bald nach dem Tod von Lola, einem Opfer des Regimes, blickt die Erzählerin in den Kühlschrank eines Essraumes und stellt sich das Bild bzw. den Gestank eines durchsichtigen Mannes vor, der „um länger zu leben, die Eigenweide gesunder Tiere gestohlen" (H 70) habe. Anschließend heißt es:

Ich sah sein Herztier. Es hing eingeschlossen in der Glühbirne. Es war gekrümmt und müde. Ich schlug den Kühlschrank zu, weil das Herztier nicht gestohlen war. Es konnte nur sein eigenes sein, es war häßlicher als die Eingeweide aller Tiere dieser Welt. (ebd.)

Man hat das Herztier, diese Wortbildung Herta Müllers, ${ }^{14}$ bereits als eine „Metapher der Wünsche, Begehren und Ängste", als ein Sinnbild sowohl für etwas „Allgemein-Menschliches“ als auch für etwas „Individuierendes“ (Patrut 2006, 203) gedeutet. Im Kontext eines Kühlschrankes aber, in dem ein durchsichtiger kranker Mensch imaginiert wird, dessen Herztier „,häßlicher als die Eigenweide aller Tiere dieser Welt" aussieht, taucht der Verdacht auf, dass es sich weniger um etwas vage angedeutetes „Allgemein-Menschliches“, als um ein Sinnbild der sozialen Verderbnis handelt, die sich in der Diktatur gleichsam der „Eigenweide“ des Menschen bemächtigt und inn widerwärtiger als die Tiere macht. Als innere Fäulnis des Menschen in der Diktatur lässt sich wohl auch Terezas Tumor begreifen, dessen fatale Entwicklung parallel zu ihrem moralischen Fall, der Konspiration mit der Securitate, läuft. Diese Bilder, die sich in einer Überganssphäre zwischen Wirklichkeit und Phantasie, Realität und Surrealität bewegen, ließen sich gleichzeitig als Beispiele für eine „Poetik des eigensinnigen Blicks“15 bei Herta Müller deuten, die eine erstarrte Wirklichkeit in Bewegung setzt und sie in eine neue, subjektive Perspektive stellt. Dieser besondere Eigensinn des subjektiven (Hin)Sehens konnte in der Ceauşescu-Ära, in der Herta Müller aufwuchs, als die soziale Wirklichkeit gleichsam unter dem Druck einer absurden Ideologie ihre Dynamik und ihre Lebensnähe verlor, eine Form des Widerstands bedeuten.

\section{$13 \mathrm{Vgl.} \mathrm{ebda.}$}

14 In der Forschung wird immer wieder auf eine Wortbildung angeblich rumänischen Ursprungs aus inimă (,Herz') und animal (,Tier') hingewiesen (z.B. Bozzi 2005, 122). Die Annahme setzt damit eine Wortverschmelzung zunächst im Rumänischen in der Form inimal voraus, aus der dann das Wort Herztier durch Spiegelübersetzung entstanden sei. Das Wort inimal wird aber im Rumänischen nicht verwendet und hat sich selbst in der rumänischen Übersetzung des Romans von 1997 nicht durchgesetzt. Der rumänische Titel ist nämlich kein Kompositum, sondern er heißt Animalul inimii, also wortwörtlich „Das Tier des Herzens“. Es bleibt immerhin festzuhalten, dass sich Herta Müller kreativ in einer Zwischenzone der Sprachen bewegt.

15 So Marlies Janz bereits 1985. Zitiert nach Eke (1991, 16). 


\section{IV.}

Ein besonderes Merkmal von Herta Müllers Gedächtnispoetik ist auch ihr Umgang mit der Sprache. Der neue, „eigensinnige“ Blick erzeugt eine neue Sprache, dies im breitesten Sinne verstanden als Spiel mit denotativen Elementen der Sprache (sogar mehrerer Sprachen), die aber in einen befremdenden, verblüffenden Kontext gestellt werden, aber auch als Bildlichkeit und (teils surreale) Metaphorik. ${ }^{16}$ Die Sprache des Romans verdeckt durch die Fiktionalisierung die reelle Sprechsituation im multilingualen Banat, wo beispielsweise im Sprechen der Deutschen dem banatschwäbischen Dialekt eine viel signifikantere Rolle zukam, als es im Roman tatsächlich der Fall ist, und die Kommunikation mit dem vom Hauptmann Pjele verkörperten Staatsapparat im Sinne des oben angesprochenen „,mononationalen Staatsverständnisses" ausschließlich auf Rumänisch erfolgt sein muss. Ein sprachliches Regionalbewusstsein wird immerhin an einer Stelle des Romans reflektiert, als im Kontext des Lesens von aus Deutschland eingeschmuggelten Büchern die „Kinderbettsprache“ mit der „Muttersprache“ (und natürlich auch mit der „Staatsprache") kontrastiert wird:

Die Bücher aus dem Sommerhaus waren ins Land geschmuggelt. Geschrieben waren sie in der Muttersprache, in der sich der Wind legte. Keine Staatssprache wie hier im Land. Aber auch keine Kinderbettsprache aus den Dörfern. In den Büchern stand die Muttersprache, aber die dörfliche Stille, die das Denken verbietet, stand in den Büchern nicht drin. (H 55)

Durch eine überraschende semantische Verbindung wird die Muttersprache, d.h. das Hochdeutsche, mit Denken, während das (Banater) Schwäbische mit dem Mangel an Reflexivität bzw. mit der „Stille" assoziiert. Herta Müller verbindet semantisch das von der Diktatur gebotene Schweigen mit dem Schwäbischen, das Lesen von deutschen Büchern hingegen mit dem Denken schlechthin, das sich seinerseits als eine Form des geistigen Widerstandes gegen die erstarrte Ideologie der Diktatur kundtut. Doch spielt das Schwäbische im Roman nicht nur die Rolle einer verschwiegenen, verbotenen Sprache, als die Sprache der Unterdrückten und Gequälten, ${ }^{17}$ sondern es schimmert im Text immer wieder durch, etwa in Form von Wörtern und Wortverbindungen wie „strabanzen“ (ungefähr: ,bummeln"), „schwäbisches Arschkappelmuster“, „,schwäbisches Kampelsackel“ oder „schwäbische Klobigkeit" (H 83). Sie sind allesamt gedächtnis- und identitätsbestimmend,

16 So wird beispielweise - durch die Erinnerungsbilder Terezas - von einer deutschen Kinderfrau erzählt, die nach Quitten roch, und von der Tereza Deutsch zu lernen hatte. Hier wird zunächst eine Assoziation mit einem gleichklingenden (vulgären) rumänischen (Schimpf)Wort eingeblendet - „Mein liebstes Wort war Futter [vgl. rum. futa], weil es meiner Sprache vögeln bedeutet“ $(H$ 176) -, um anschließend die Härte der beschworenen Obstsorte (als eines Denotativs) metaphorisch-surreal mit dem Deutschlernen zu verbinden: „Deutsch blieb für mich immer eine harte Quittensprache.“ (H 177)

17 Mag sein, dass das Schwäbische in anderen Werken wie Niederungen oder in Essays wie Der Teufel sitzt im Spiegel oder Das Ticken der Norm eine andere Rolle in Herta Müllers Werk spielt, doch ist im vorliegenden Roman die Antithese zu einem rückständigen, bedrückenden Dorfmilieu weniger prägnant. 
sprachliche Reminiszenzen einer Identitätsform, die trotz permanenter Gefährdungen und Drohungen von den Rumäniendeutschen nie aufgegeben wurde.

Müllers Neigung zur Sprachmischung, ihre häufig beschworene Polyglossie (etwa Anițaş 2017) ist ihrerseits ein spezifisches Bauelement der Identitätsbildung im Roman. Der Text erhält durch die Verwendung ungarischer (gelegentlich auch schwäbischer und rumänischer) Sprachelemente eine interkulturelle Färbung. Die Mischung von Wörtern aus verschiedenen, im Banat gesprochenen Sprachen oder Dialekten zeichnet einen interkulturellen Raum nach und erweitert das semantische Deutungsfeld der einzelnen Kontexte. Bezeichnend hierfür sind die Beschreibungen der Frau Margit, einer „Ungarin aus Pest“, die zwar eine rege Kirchgängerin ist, sich aber im Zorn leicht zum Fluchen hinreißen lässt:

Nur wenn Frau Margit im Zorn die Kartoffeln, die sie später schälte, aus der Kiste an die Wände schmiß, vergaß sie ihren Jesus und fluchte ungarisch. Wenn die Kartoffeln gekocht auf dem Tisch standen, küßte sie an der Stelle, wo Jesus das Tuch trug, alle Flüche wieder weg. (H 130)

Mit der Sprache von Margit rückt die Vielsprachigkeit als alltägliche Praxis in den Mittelpunkt, indem anhand einiger Episoden dem Leser die Selbstverständlichkeit interkulturellen Sprachgebrauchs und Handelns vorgeführt wird, die für eine plurikulturelle Region wie das Banat so typisch war. Frau Margit fragt, „wo a fene“ (,wo zum Teufel') die „Fetzen“ (Lappen) liegen, sie deklamiert, sie wolle „keine kurva [Hure] im Haus", oder präsentiert ihre auf Stereotypen gegründeten Vorstellungen: „nur gazember [Schurken] haben rote Haare“ (H 133).

Solche fremdsprachlichen Einschübe entspannen stellenweise die sonst sehr intensive, dramatische Prosa Herta Müllers. Sie spiegeln aber gleichzeitig das Sprach- und Identitätsbewusstsein der Bewohner eines multiethnischen (kulturell aber mehr oder weniger einheitlichen) Raumes wider.

Doch damit erschöpft sich das Spektrum des Erzählten nicht, denn die von Herta Müller am Ende der achtziger Jahre erlebte Auswanderung nach Deutschland, das Schicksal von zehntausenden Rumäniendeutschen, wird auf den letzten Seiten des Romans ebenfalls thematisiert. Die Ankunft in Deutschland wird dabei nicht idealisiert, sondern vielmehr in einem nüchternen Ton problematisiert, was die Identitäts- bzw. Zugehörigkeitsfrage um eine weitere Dimension erweitert. Allerdings wird das Identitätsproblem wie auch die deutsche Vergangenheit und Gegenwart in einer Reihe von eindrucksvollen Sätzen bzw. Erinnerungsbildern lediglich angedeutet, sodass der Deutungsprozess im Grunde dem Leser überlassen wird. Tangiert werden aber die Nazi-Vergangenheit mit einem Hinweis auf die Lieder des Vaters „für den Führer“, die Heimkehr der SS-Soldaten, bzw. der Identitätsfaktor „Mutterland“ im Kontext einer konzis skizzierten Familiengeschichte: „Der Großvater, der Frisör, der Uhrmachertoni, der Vater, der Pfarrer und Lehrer nannten Deutschland das Mutterland." Gleichzeitig wird die Gültigkeit des emotionalen Raumbezugs anhand von Begriffen wie „Deutschland“ oder „Mutterland“ ironisch relativiert und damit wieder demontiert: „Obwohl Väter für Deutschland in die Welt marschiert waren, war es das Mutterland." (H 238) 
Das Bild des „Mutterlandes“ wird indessen auch dadurch relativiert, dass wegen der (bürokratisch-politischen) Schwierigkeiten der Übersiedlung, der telefonischen Todesdrohungen aus Rumänien, des heuchlerischen Spiels der falschen Freundin Tereza, der Todeserinnerungen (Begräbnis der Großmutter) und Todesnachrichten (von Kurts Selbstmord) jede Spur der heimatlichen Geborgenheit verloren geht. Die Migrantin schwebt in einem Zwischenzustand zwischen einem Nicht-mehr und einem Noch-nicht: Die Aussiedlung nach Deutschland erscheint in Roman weder als eine euphorische Entdeckung noch als ein abrupter Bruch mit der Banater Vergangenheit. Trotz der individuellen Perspektive wird darin das Schicksal einer Gemeinschaft artikuliert. Müllers Herztier ist ein besonderes literarisches Denkmal der Geschichte der Rumäniendeutschen aus den 1970er- und 1980er-Jahren und gleichzeitig ein bleibendes Zeugnis der Meisterschaft deutschsprachigen Schreibens um die Jahrtausendwende.

\section{Literaturverzeichnis}

Anițaş, Tünde Éva (2017): Die Mehrsprachigkeit in den Werken von Herta Müller. Hamburg: Dr. Kovač.

Bergel, Hans (1995): Erkundungen und Erkennungen. Notizen eines Neugierigen. München: Süddeutsches Kulturwerk.

Bozzi, Paola (2005): Der fremde Blick. Zum Werk Herta Müllers. Würzburg: Königshausen \& Neumann.

Eke, Norbert Otto (1991): Augen/Blicke oder: Die Wahrnehmung der Welt in den Bildern. Annäherung an Herta Müller. In Die erfundene Wahrnehmung. Annäherung an Herta Müller. Eke, Norbert Otto. Paderborn: Igel, 7-21. p.

Engler, Jürgen (1995): Erfahrung, leibhaft. Herta Müller: „Herztier“. In Neue deutsche Literatur 43/499, 173-176. p.

Grotzky, Johannes (2019): Rumänien - Untergang einer Diktatur: Umsturz und Machtkampf. Reportagen 1989/1990. Norderstedt: BoD.

Höhne, Steffen (2014): Das Banat (Bánság) als kulturhistorische Transferregion. Literarisch-kulturelle Konstitutionsprozesse in Zentraleuropa. In Kann Literatur Zeuge sein? Poetologische und politische Aspekte in Herta Müllers Werk. Jahrbuch für internationale Germanistik. Dorle Mérchiers, Jacques Lajjarige u. Steffen Höhne (Hrsg.). Bern u.a.: Peter Lang, 17-32. p.

Kimmerle, Heinz (1994): Die Dimension des Interkulturellen. Philosophie in Afrika afrikanische Philosophie. Zweiter Teil: Supplemente und Verallgemeinerungsschritte. Amsterdam/Atlanta: Rodopi.

Müller, Herta (2009): Herztier. 2. Aufl. Frankfurt a.M.: Fischer $(=\mathrm{H})$

Müller, Herta (2011): Immer derselbe Schnee und immer derselbe Onkel. München: Hanser.

Müller, Herta (2013): Herzwort und Kopfwort. In Spiegel Online vom 21.01.2013. http:// www.spiegel.de/spiegel/print/d-90638332.html. 
Nubert, Roxana (2014): Die Diktatur im Spiegel der Literatur: Surreale Bildlichkeit in Herta Müllers Romanen Herztier und Heute wäre ich mir lieber nicht begegnet. In Kann Literatur Zeuge sein? Poetologische und politische Aspekte in Herta Müllers Werk. Jahrbuch für internationale Germanistik. Dorle Mérchiers, Jacques Lajjarige u. Steffen Höhne (Hrsg.). Bern u.a.: Peter Lang, 217-235. p.

Patrut, lulia-Karin (2006): Schwarze Schwester - Teufelsjunge. Ethnizität und Geschlecht bei Paul Celan und Herta Müller. Köln/Weimar: Böhlau.

Wirth, Uwe (2012): Zwischenräumliche Bewegungspraktiken. In Bewegen im Zwischenraum. Uwe Wirth. (Hrsg.). Berlin: Kadmos, 7-34. p. 